资

\title{
Effects of bariatric surgery on urinary incontinence
}

\section{Efeitos da cirurgia bariátrica na incontinência urinária}

\author{
Mayra Jaqueline Rocha ${ }^{1}$, Rafael Luiz Dias ${ }^{1 *}$, Lizyana Vieira ${ }^{2}$ \\ ${ }^{1}$ Acadêmicos do curso de Fisioterapia do Centro Universitário Fundação Assis Gurgacz, Cascavel - PR. \\ ${ }^{2}$ Fisioterapeuta, Mestre, Docente do curso de Fisioterapia do Centro Universitário Fundação Assis Gurgacz, Cascavel \\ - PR.
}

\section{ARTICLE INFO}

Article history:

Received 10 September 2020

Revised 19 October 2020

Accepted 21 November 2020

Available online 18 December 2020

Blind reviews

\section{Keywords: \\ Obesity \\ Weight LosS}

Gastrectomy

\section{Palavras-chave:}

Obesidade

Perda de peso

Gastrectomia

* Corresponding author at: rafael_dias13@hotmail.com https://orcid.org/0000-0002-1723-1417

\begin{abstract}
Introduction: Obesity is considered by the World Health Organization (WHO) as a worldwide epidemic in developed and developing countries. Urinary incontinence is one of the pelvic floor disorders common in the obese population and a worldwide health problem. Objective: To evaluate, through a systematic review, the relationship between the effects of bariatric surgery on urinary incontinence in an obese population. Methodology: This article is a systematic review of an observational and perspective cohort study. Searched in the sources: Medline, Lilacs, Bireme, and Scielo. Results: A total of 84 articles were found in the records identified through searches in the electronic databases. Of these, 23 articles were selected for eligibility for analysis of the abstract and methodology. 12 articles were excluded for not meeting the prerequisites. After analyzing the PEDro scale, 8 articles were selected to compose this study. Conclusion: This review allowed us to identify that weight loss induced by bariatric surgery positively influences pelvic floor disorders, especially in the reduction or resolution of urinary incontinence symptoms, which consequently improves quality of life.
\end{abstract}




\section{Introdução}

A obesidade é considerada pela Organização Mundial da Saúde (OMS) como uma epidemia mundial em países desenvolvidos e em desenvolvimento (HERDY et al, 2014). No Brasil, a ampliação dos casos de obesidade e sobrepeso ocorre em todas as idades, ambos os sexos e classes sociais, sendo mais rápido o aumento de casos em famílias com menor rendimento. Referente à incidência de obesidade e sobrepeso no Brasil, o Instituto Brasileiro de Geografia e Estatística (IBGE) realizou uma pesquisa em 2013, onde apontou que $56,9 \%$ da população adulta está com sobrepeso, e 20,8\% são obesas (DIAS et al., 2017).

A OMS define obesidade como acúmulo anormal ou excessivo de gordura corporal em forma de tecido adiposo e é considerada uma doença de causa multifatorial, envolvendo fatores genéticos, metabólicos, ambientais e comportamentais. A obesidade aumenta o risco do indivíduo desenvolver patologias crônicas como doenças cardiovasculares, diabetes mellitus tipo II, osteoartrite, dislipidemias, entre vários outros (TONATTO et al., 2019).

Além das alterações pressupostas, a obesidade leva a um aumento crônico da pressão intra-abdominal, enfraquecendo os músculos do diafragma da pelve, aumentando as chances da pessoa obesa desenvolver distúrbios do assoalho pélvico, como incontinência urinária (CASTRO et al., 2012).

A incontinência urinária (IU) é uma das desordens do assoalho pélvico comum na população obesa e um problema de saúde mundial, sendo definida pela International Continence Society (ICS) como qualquer perda involuntária de urina (ANGLIM et al., 2018). A incontinência urinária é considerada um problema de saúde pública, e essa condição afeta diretamente a qualidade de vida do indivíduo, e ainda assim, muitas vezes acaba sendo abordada sem a devida importância, sendo frequentemente subtratada (ROCHA et al., 2017).

A IU é classificada em três tipos principais: a Incontinência Urinária de Esforço (IUE), quando ocorre perda de urina durante algum esforço que aumente a pressão intraabdominal, como tosse, espirro ou exercícios físicos; urgeincontinência ou Incontinência Urinária de Urgência (IUU), caracterizada pela perda de urina acompanhada de forte sensação de urgência para urinar; e a Incontinência Urinária Mista (IUM), quando há queixa de perda associada à urgência e também a esforços (MELO et al., 2012).

Uma opção mais rápida para o tratamento da obesidade mórbida e seus efeitos é a cirurgia bariátrica. A principal técnica utilizada é o bypass gástrico, que causa uma redução na cavidade gástrica e na quantidade de alimentos ingeridos, devido à restrição e redução da superfície de absorção intestinal. Essas alterações provocam além da perda ponderal, o aumento da saciedade, induzindo a perda de peso (TONATTO et al., 2019).

Sendo assim, o objetivo desse estudo foi avaliar, através de uma revisão sistemática, a relação dos efeitos da cirurgia bariátrica na incontinência urinária em população obesa.

\section{Metodologia}

Para o cumprimento deste trabalho foi realizada uma revisão sistemática de estudos de coorte observacional e prospectivo. Foram utilizados estudos que tiveram como objetivo avaliar a influência da cirurgia bariátrica na incontinência urinária de indivíduos obesos.
Foram incluídos no estudo ensaios clínicos nacionais e internacionais recentes, de 10 anos de publicação, buscados nas fontes: MEDLINE (Medical Literature Analysis and Retrieval System Online), LILACS (Literatura Latinoamericana e do Caribe em Ciências da Saúde), BIREME (Centro Latino-Americano e do Caribe de Informação em Ciências da Saúde), SCIELO. Para realizar a busca foram utilizados os descritores em saúde "Obesity", "Weight Loss", "Pelvic Floor Disorders", "Pelvic Floor" e "Gastrectomy".

A busca por referências foi realizada nos meses de maio a junho de 2020. Os artigos passaram por uma pré análise onde foram selecionados a partir do título, resumo e metodologia, seguindo os critérios de inclusão: artigo científicos com até 10 anos de publicação; artigos científicos publicados em língua portuguesa e inglesa. Após a seleção, cada autor realizou avaliação qualitativa dos artigos na escala PEDro de forma individual, e posteriormente foi realizada a conferência das pontuações em conjunto. Foram excluídos deste estudo artigos científicos de revisão bibliográfica; resumos de artigos científicos para eventos; estudo de caso; nota menor que 3 na escala PEDro.

\section{Análise e discussão dos resultados}

Um total de 84 artigos foram encontrados nos registros identificados através das buscas nas bases de dados eletrônicas. Destes, 23 artigos foram selecionados por elegibilidade para análise do resumo e metodologia. Foram excluídos 12 artigos por não cumprirem os pré-requisitos. Após a análise na escala PEDro 8 artigos foram selecionados para compor este estudo.

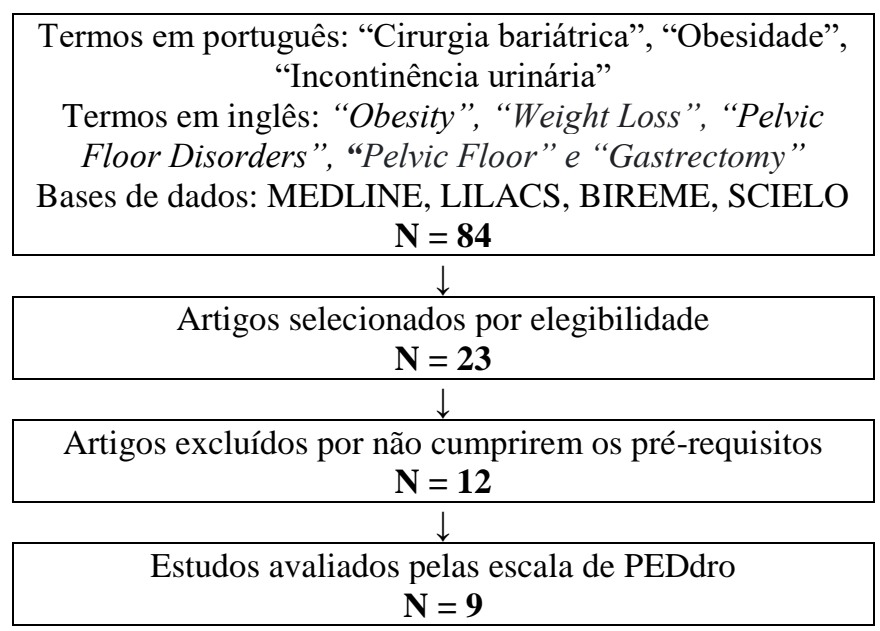

Figura 1 - Etapas do processo de seleção dos artigos.

De acordo com a análise dos artigos selecionados o tipo de incontinência urinária mais frequente foi a incontinência urinária de esforço (IUE). Além disso, a obesidade provoca efeitos crônicos prejudiciais na IU. Indivíduos que são obesos por mais de 30 anos tem maior predisposição a ter IUE em comparação com obesos há um período mais curto de 5 a 10 anos. (ANGLIM et al., 2018).

No estudo de Ait Said et al. (2016), os pacientes selecionaram a técnica melhor adaptada para a cirurgia de redução de peso optando entre sleeve e bypass gástrico. Ao final do estudo foi observado redução significativa na incontinência urinária de urgência e esforço, além da disúria e melhora na qualidade de vida após a perda de peso.

FAG Journal of Health - ISSN 2674-550X, 2020, v.2, n.4, p.467 
Quadro 1. Características e resultados dos artigos incluídos neste estudo

\begin{tabular}{|c|c|c|c|c|c|c|}
\hline $\begin{array}{c}\text { Autor, } \\
\text { ano }\end{array}$ & Título & PEDro & Estudo & Objetivo & Amostra & Resultados \\
\hline $\begin{array}{l}\text { Ait Said et } \\
\text { al., } 2016\end{array}$ & $\begin{array}{l}\text { Effect of bariatric } \\
\text { surgery on urinary and } \\
\text { fecal incontinence: } \\
\text { prospective } \\
\text { analysis with 1-year } \\
\text { follow-up }\end{array}$ & 5 & $\begin{array}{l}\text { Estudo de } \\
\text { coorte } \\
\text { observacional }\end{array}$ & $\begin{array}{lrr}\text { Determinar } & \text { o efeito } & \text { da } \\
\text { cirurgia } & \text { bariátrica } & \text { na } \\
\text { incontinência } & \text { urinária, } \\
\text { disúria e incontinência fecal } \\
\text { antes } & \text { e } & 1 \\
\text { ano após } & \text { a } & \text { cirurgia } \\
\text { bariátrica. } & & \\
\end{array}$ & $\begin{array}{l}140 \\
\text { pacientes }\end{array}$ & $\begin{array}{l}\text { A taxa de incontinência } \\
\text { urinária de esforço e de } \\
\text { urgência apresentou } \\
\text { redução significativa } \\
\text { após } 1 \text { ano da cirurgia } \\
\text { bariátrica. }\end{array}$ \\
\hline $\begin{array}{l}\text { Anglin et } \\
\text { al., } 2018\end{array}$ & $\begin{array}{l}\text { The long-term effects } \\
\text { of bariatric surgery on } \\
\text { female urinary } \\
\text { incontinence }\end{array}$ & 3 & $\begin{array}{l}\text { Estudo de } \\
\text { coorte } \\
\text { prospectivo }\end{array}$ & $\begin{array}{l}\text { Determinar o impacto da } \\
\text { cirurgia bariátrica na IU } \\
\text { feminina em doze meses } \\
\text { pós-operatório. }\end{array}$ & $\begin{array}{l}366 \\
\text { mulheres }\end{array}$ & $\begin{array}{l}\text { A redução } \text { de } \\
\text { Incontinência urinária } \\
\text { de esforço, bexiga } \\
\text { hiperativa e mista foi de } \\
41 \%, \quad 38 \% \text { e } 48 \% \\
\text { respectivamente. }\end{array}$ \\
\hline $\begin{array}{l}\text { Castro et } \\
\text { al., } 2012\end{array}$ & $\begin{array}{l}\text { Effects of bariatric } \\
\text { surgery on pelvic floor } \\
\text { function }\end{array}$ & 3 & $\begin{array}{l}\text { Estudo de } \\
\text { coorte } \\
\text { observacional }\end{array}$ & $\begin{array}{l}\text { Avaliar os efeitos da cirurgia } \\
\text { bariátrica na função } \\
\text { assoalho do } \\
\text { mulheres. }\end{array}$ & $\begin{array}{c}30 \\
\text { mulheres }\end{array}$ & $\begin{array}{l}\text { A prevalência de } \\
\text { incontinência urinária } \\
\text { passou de } 70,8 \% \text { no pré- } \\
\text { operatório para } 20,8 \% \\
\text { no pós-operatório. }\end{array}$ \\
\hline $\begin{array}{l}\text { Defoor et } \\
\text { al., } 2017\end{array}$ & $\begin{array}{l}\text { Prospective evaluation } \\
\text { of urinary incontinence } \\
\text { in severely obese } \\
\text { adolescents presenting } \\
\text { for weight loss surgery }\end{array}$ & 3 & $\begin{array}{l}\text { Estudo de } \\
\text { coorte } \\
\text { prospectivo }\end{array}$ & $\begin{array}{lr}\text { Avaliar os } & \text { sintomas } \\
\text { incontinência } & \text { em } \\
\text { adolescentes } & \text { gravemente } \\
\text { obesos antes e } 3 \text { anos após a } \\
\text { cirurgia bariátrica. }\end{array}$ & $\begin{array}{l}242 \\
\text { pacientes }\end{array}$ & $\begin{array}{l}\text { A prevalência de IU em } \\
\text { mulheres e homens } \\
\text { diminuiu para } 7 \% \text { e } 0 \% \text {, } \\
\text { respectivamente, aos } 6 \\
\text { meses após a cirurgia. }\end{array}$ \\
\hline $\begin{array}{l}\text { Gabriel at } \\
\text { al., } 2018\end{array}$ & $\begin{array}{lr}\text { Pelvic } & \text { Organ } \\
\text { and Prolapse } \\
\text { Incontinence } & \text { Urinary } \\
\text { Women After } & \text { Bariatric } \\
\text { Surgery: } & \text { 5-Year } \\
\text { Follow-up } & \\
\end{array}$ & 6 & $\begin{array}{l}\text { Estudo } \\
\text { coorte }\end{array}$ & $\begin{array}{l}\text { O objetivo foi determinar se } \\
\text { a cirurgia bariátrica está } \\
\text { associada à diminuição da } \\
\text { prevalência de sintomas de } \\
\text { incontinência urinária e } \\
\text { prolapso de órgãos pélvicos. }\end{array}$ & $\begin{array}{l}388 \\
\text { mulheres }\end{array}$ & $\begin{array}{l}\text { Das } 91 \text { mulheres com IU } \\
\text { antes da } \quad \text { cirurgia } \\
\text { bariátrica, } \\
\text { relataram diminuição } \\
\text { dos sintomas }\end{array}$ \\
\hline $\begin{array}{l}\text { Knepfler } \\
\text { et al., } \\
2015\end{array}$ & $\begin{array}{l}\text { Bariatric surgery } \\
\text { improves female pelvic } \\
\text { floor disorders }\end{array}$ & 3 & $\begin{array}{l}\text { Estudo de } \\
\text { coorte } \\
\text { prospectivo }\end{array}$ & $\begin{array}{l}\text { Avaliar a evolução das } \\
\text { doenças do assoalho pélvico } \\
\text { em mulheres que se } \\
\text { submeteram à cirurgia } \\
\text { bariátrica. }\end{array}$ & $\begin{array}{c}70 \\
\text { pacientes }\end{array}$ & $\begin{array}{l}\text { Houve redução } \\
\text { significativa no índice } \\
\text { de incontinência } \\
\text { urinária após } 11 \text { meses } \\
\text { de cirurgia bariátrica. } \\
\end{array}$ \\
\hline $\begin{array}{l}\text { Leshem et } \\
\text { al., } 2018\end{array}$ & $\begin{array}{l}\text { Surgically induced } \\
\text { weight loss results in a } \\
\text { rapid and consistent } \\
\text { improvement of female } \\
\text { pelvic floor symptoms }\end{array}$ & 3 & $\begin{array}{l}\text { Estudo de } \\
\text { coorte } \\
\text { observacional }\end{array}$ & $\begin{array}{l}\text { O objetivo foi avaliar o } \\
\text { efeito ao longo do tempo da } \\
\text { cirurgia bariátrica nos } \\
\text { sintomas do assoalho } \\
\text { pélvico feminino. }\end{array}$ & $\begin{array}{l}101 \\
\text { mulheres }\end{array}$ & 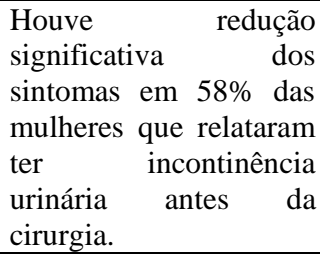 \\
\hline
\end{tabular}

Fonte: Autor

No estudo de Gabriel et al. (2018), os pacientes foram submetidos a uma das seguintes técnicas de cirurgia bariátrica: banda gástrica ajustável, bypass gástrico, ou sleeve. Foram avaliadas 388 mulheres com um questionário de autorrelato incluindo perguntas referentes à IU, prolapso e incontinência fecal. Das mulheres avaliadas apenas 91 apresentaram IU antes da cirurgia bariátrica, destas $46 \%$ relataram redução dos sintomas após a cirurgia.

Leshem et al (2018), avaliaram 101 mulheres que foram submetidas a cirurgia bariátrica utilizando o método bypass gástrico ou gastrectomia laparoscópica, utilizando o questionário "International Consultation on Incontinence Questionnaire - Short Form” (ICIQ-SF). A avaliação foi realizada em 5 etapas: no pré-operatório, 3, 6, 12 e 24 meses após a cirurgia. Das mulheres avaliadas 43 apresentaram IU no pré-operatório, destas $63 \%$ relataram IUE, 28\% IUM, e 4,5\% IUU. Houve redução significativa dos sintomas em $58 \%$ das mulheres que relataram ter sintomas de IU antes da cirurgia.

Whitcomb et al (2012) avaliaram 98 mulheres, sendo 95 submetidas ao procedimento de banda gástrica ajustável e 3 ao sleeve. As mulheres foram avaliadas através do questionário "Epidemiology of Prolapse and Incontinence Questionnaire” (EPIQ) em 3 momentos, no pré operatório, 6 e 12 meses após a cirurgia. Após avaliação no pré-operatório 22 mulheres apresentaram IUE, destas $48 \%$ tiveram resolução dos sintomas de IUE após a cirurgia.

Knepfler et al (2015), avaliaram mulheres obesas através do questionário "Pelvic Floor Distress Inventory" (PFDI-20). As pacientes realizaram cirurgia bariátrica utilizando o método bypass gástrico ou sleeve. O questionário foi aplicado no pré-operatório, no pós-operatório de 1 mês, e depois disso, em intervalos de 6 meses. Após a comparação dos resultados foi constatado que houve redução significativa de incontinência urinária após a realização da cirurgia, sendo que de $81 \%$ das mulheres afetadas no pré-operatório passou para $59 \%$.

Anglim et al. (2018) e Castro et al. (2012) avaliaram a presença de IU em obesos em 2 momentos: no pré-operatório e 1 ano após a cirurgia bariátrica, porém com objetivos diferentes. Anglim et al utilizaram como ferramenta de avaliação o questionário "International Consultation on Incontinence Questionnaire - Short Form” (ICIQ-SF) no FAG Journal of Health - ISSN 2674-550X, 2020, v.2, n.4, p.468 
intuito de observar a prevalência de IU. Já Castro et al utilizaram para avaliação o "King's health Questionnaire" para observar o impacto da IU na qualidade de vida. No estudo de Anglim et al 366 pacientes preencheram o questionário e 151 relataram ter IUM, IUE ou bexiga hiperativa no préoperatório. Após a cirurgia houve redução na prevalência de IUM em $48 \%$, IUE em $41 \%$ e bexiga hiperativa em $38 \%$. Castro et al avaliaram em 30 mulheres a prevalência de incontinência urinaria no pré-operatório, a qual passou de $70,8 \%$ no pré-operatório para $20,8 \%$ no pós-operatório, concluindo que a cirurgia bariátrica repercute positivamente na IU e na qualidade de vida de mulheres com obesidade mórbida.

Defoor et al. (2017), em seu estudo comprovaram que a prevalência de IU em obesos diminuiu significativamente após 6 meses de cirurgia, apresentando evidências que a cirurgia bariátrica interfere positivamente nos mecanismos anatômicos e fisiológicos do sistema urinário em indivíduos obesos.

\section{Considerações finais}

Esta revisão nos permitiu identificar que a perda ponderal induzida por cirurgia bariátrica influencia positivamente nos distúrbios do assoalho pélvico, especialmente na diminuição ou resolução dos sintomas de incontinência urinária, o que consequentemente melhora a qualidade de vida.

\section{Conflito de interesse}

Os autores afirmam não haver conflito de interesse.

\section{Referências}

AIT SAID, K. et al. Effect of bariatric surgery on urinary and fecal incontinence: prospective analysis with 1-year follow-up. Surgery for Obesity and Related Diseases, v. 13, n. 2, p. 305312, 2017. https://doi.org/10.1016/j.soard.2016.08.019

ANGLIM, B. et al. The long-term effects of bariatric surgery on female urinary incontinence. European Journal of Obstetrics and Gynecology and Reproductive Biology, v. 231, p. 15-18, 2018. https://doi.org/10.1016/j.ejogrb.2018.10.011

CASTRO, L. A. DE et al. Efeitos da cirurgia bariátrica na função do assoalho pélvico. ABCD. Arquivos Brasileiros de Cirurgia Digestiva (São Paulo), v. 25, n. 4, p. 263-268, 2012. https://doi.org/10.1590/S0102-67202012000400010

DEFOOR, W. R. et al. Prospective evaluation of urinary incontinence in severely obese adolescents presenting for weight loss surgery. Surgery for Obesity and Related Diseases, v. 14, n. 2, p. 214 218, 2018. https://doi.org/10.1016/j.soard.2017.09.510

DIAS, P. C. et al. Obesity and public policies: The Brazilian government's definitions and strategies. Cadernos de Saude Publica, v. 33, n. 7, p. 1-12, 2017. https://doi.org/10.1590/0102$\underline{311 \times 00006016}$

GABRIEL et al. Pelvic Organ Prolapse and Urinary Incontinence in Women After Bariatric Surgery: 5-Year Follow-up Iwona. Female Pelvic Medicine \& Reconstructive Surgery, v. 26, n. 5, p. 287-298, 2020. https://www.doi.org/10.1007/s00192-0121756-5

HERDY et al. Brazilian Guidelines for cardiovascular prevention and rehabilitation. Arquivos Brasileiros de Cardiologia, v. 103, n. 2, 2014. https://doi.org/10.5935/abc.2014S003

KNEPFLER, T. et al. Bariatric surgery improves female pelvic floor disorders. Journal of Visceral Surgery, v. 153, n. 2, p. 95-99, 2016. http://dx.doi.org/10.1016/j.jviscsurg.2015.11.011

LESHEM, A. et al. Surgically induced weight loss results in a rapid and consistent improvement of female pelvic floor symptoms. Scandinavian Journal of Urology, v. 52, n. 3, p. 219-224, 2018 https://doi.org/10.1016/j.ejogrb.2018.10.011
TONATTO-FILHO, A. J. et al. Cirurgia Bariátrica No Sistema Público De Saúde Brasileiro: O Bom, O Mau E O Feio, Ou Um Longo Caminho A Percorrer.Sinal Amarelo! Arquivos Brasileiros De Cirurgia Digestiva, v. 32, n. 4, p. 1-5, 2019. http://dx.doi.org/10.1590/0102-672020190001e1470

WHITCOMB, E. L. et al. Impact of surgically induced weight loss on pelvic floor disorders. International Urogynecology Journal, v. 23, n. $\quad 8, \quad$ p. $1111-1116, \quad 2012$. https://www.doi.org/10.1007/s00192-012-1756-5 